\title{
Functional implications of neuropsychological normality and symptom remission in older outpatients diagnosed with schizophrenia: A cross-sectional study
}

\author{
WINNIE W. LEUNG, ${ }^{1,2}$ CHRISTOPHER R. BOWIE, ${ }^{2,3}$ AND PHILIP D. HARVEY ${ }^{2,4}$ \\ ${ }^{1}$ Department of Veterans Affairs, VISN 3 Mental Illness, Research, Education and Clinical Center (MIRECC), Bronx, New York \\ ${ }^{2}$ Department of Psychiatry, Mt. Sinai School of Medicine, New York, New York \\ ${ }^{3}$ James J. Peters Bronx VA Medical Center, Bronx, New York \\ ${ }^{4}$ Department of Psychiatry, Emory University School of Medicine, Atlanta, Georgia \\ (Received June 22, 2007; Final Revision February 1, 2008; Accepted February 1, 2008)
}

\begin{abstract}
Cognitive impairments in schizophrenia are well documented and correlated with functional disability. Although some patients demonstrate normal neuropsychological (NP) functioning, little is known about their functional disability. We examined the cross-sectional functional implications of NP normality and symptomatic remission in older outpatients diagnosed with schizophrenia or schizoaffective disorder, who were administered a NP battery and performance-based measures of functional and social competence, with their real-world functioning rated by case managers. NP status was classified by the General Deficit Score (GDS) and remission status was based on the Positive and Negative Syndrome Scale (PANSS), yielding four subsamples of patients: NP normal-remitted $(n=$ $21)$, NP normal-symptomatic $(n=22)$, NP impaired-remitted $(n=90)$, and NP impaired-symptomatic $(n=97)$. NP normal patients demonstrated better functional and social competence and better ratings of real world functioning, after controlling for premorbid abilities. However, compared to normative date, NP normal patients manifested disability in several real-world domains, including residential status. These results suggest that NP status is a better predictor of functional outcome then symptom status or the interaction of the two factors. The disability seen in NP normal cases indicates that factors other than cognitive impairments may determine aspects of everyday outcomes in schizophrenia. (JINS, 2008, 14, 479-488.)
\end{abstract}

Keywords: Cognition, Normal range, Outcome, Adjustment, Social behavior, Independent living

\section{INTRODUCTION}

Significant functional disability is found in most patients diagnosed with schizophrenia in younger (Green et al., 2000) and older samples (Palmer et al., 2002), yet disability remains an elusive treatment target. Despite demonstrated efficacy for positive symptoms, antipsychotic medications have not effectively promoted truly independent living, return to the workforce, or establishment and maintenance of social relationships (Mueser, 2000). Impairments in social and occupational functioning are usually present at the onset of the illness and tend to remain even

Correspondence and reprint requests to: Winnie W. Leung, Ph.D., Lieber Center for Schizophrenia Research and Treatment, New York State Psychiatric Institute, 1051 Riverside Drive, Unit 14, New York, NY 10032. E-mail: leungwi@pi.cpmc.columbia.edu after treatment is initiated (Hafner \& an der Heiden, 2003), implicating non-symptomatic causes for these impairments.

Evidence from cross-sectional (Green, 1996; Harvey et al., 1998) and longitudinal studies (Green et al., 2004) has consistently shown cognitive impairment in schizophrenia to be a more stable and robust correlate of functional impairment than clinical symptoms. The correlations between impairments in neuropsychological (NP) performance and impairments in everyday living skills are found across a wide range of skills (Evans et al., 2003). For example, patients with more cognitive impairments have greater impairments in social functioning (Smith et al., 1999), are less likely to have successful social relationships (Bowie et al., 2006), and are less likely to live independently (Twamley et al., 2002). Given these findings, pharmacological (Harvey et al., 2003, 2004; Keefe et al., 2004) and 
behavioral interventions (Hogarty et al., 2004; McGurk et al., 2005; Wexler \& Bell, 2005) have targeted cognition, which has been designated as an urgent treatment priority (Marder \& Fenton, 2004).

Although the breadth and severity of cognitive impairment in schizophrenia is well documented, the overall degree of impairment varies greatly across patients (Goldstein, 1994). Many studies have found a small subgroup of patients whose performance puts them within the normal range of NP functioning. The prevalence of NP normality, however, has been variable across studies, ranging from $0 \%$ (Bozikas et al., 2006; Wilk et al., 2005) to 82\% (Torrey et al., 1994), with the majority of studies reporting rates closer to $25 \%$ (Bryson et al., 1993; Kremen et al., 2000; Palmer et al., 1997; Rund et al., 2006). These discrepancies are likely caused by sample characteristics and how normality is operationalized. Previous studies have varied in that they defined normal as having one or fewer ability areas performed at less than -1 SD compared to healthy controls (HC) (Palmer et al., 1997), having fewer than two abilities performed at less than -2 SD compared to HC (Kremen et al., 2000), and having all NP abilities performed at levels consistent with IQ scores (Wilk et al., 2005).

Despite the extensive research on the prevalence of NPnormality, very little is known about the everyday functions of this subgroup of individuals diagnosed with schizophrenia. The functional implications of having schizophrenia and NP normality would have direct relevance to treatment goals. Because the ultimate aim of cognitive enhancement is to support functional recovery, cases where NP performance is normal may have minimal intervention potential. The relationship between cognitive dysfunction and everyday behavior is complex, dependent in part on competencies in the areas of selfcare and social abilities and mediated and limited by the severity of depression and negative symptoms (Bowie et al., 2006; in press) as well as social factors such as disability payment status and ethnic variation (Rosenheck et al., 2006). Thus, it is possible that patients with normal NP performance would still manifest some evidence of real-world functional disability caused by these other factors.

A recent development in the clinical assessment of schizophrenia is the application of the clinical remission concept, widely employed in depression (Frank et al., 1991) and bipolar disorder (Nuechterlein et al., 2006), to schizophrenia. Formal criteria for remission have been developed and several studies have examined the prevalence and correlates of clinical remission (e.g., Andreasen et al., 2005; De Hert et al., 2007; van Os et al., 2006). Because positive symptoms have been found to be quite poorly correlated on a cross-sectional basis with the severity of NP impairment and functional disability, it is unclear what the functional implications of cross-sectional clinical remission would be. There are recent indications that sustained remission has important functional implications (De Hert et al., 2007), possibly related to previous findings suggesting that patients with particularly persistent psychotic symptoms have increased functional disability (e.g., Keefe et al., 1996).
In the current study, we examined the functional outcome of neuropsychological normality and cross-sectional symptomatic remission in schizophrenia using performance-based measures of functional capacity as well as ratings of everyday functioning indexed by case manager reports. The goal of this investigation is to examine whether neuropsychological and cross-sectional symptomatic status differentially predicted elements of functional outcome. That is, rather than testing the concept of normality, this study aims to examine the clinical relevance of normality by using a classification system that has shown good convergent validity in the literature (Carey et al., 2004). We selected performance-based measures of everyday functioning because they capture the actual ability of the patient to perform everyday skills and may be more suitable than real-world achievements to capture changes (Buchanan et al., 2005) in short term studies. For instance, antipsychotic treatment-related changes in social skills performance as measured by the Social Skills Performance Assessment have been shown to be correlated with concurrent improvements in neuropsychological performance (Harvey et al., 2006).

Patients were classified as NP normal or NP impaired based on the Global Deficit Score (GDS; Carey et al., 2004) classification system and compared on measures of functional competence, including both everyday living skills and social abilities, and real world outcomes. The term NP normality in this study does not necessarily signify a lack of decline from premorbid functioning, but rather, it is defined by performance that is within normal limits. Symptom remission, as determined by the Andreasen et al. (2005) criteria for the Positive and Negative Symptom Scale (PANSS; Kay et al., 1987), was also examined crosssectionally to determine its relationships with functional outcomes, in addition to NP normality. We also examined the prevalence of several major milestones, including marriage, employment, and independence in residential status, in patients with and without NP normality and symptomatic remission.

\section{METHODS}

\section{Subjects}

Older community dwelling outpatients who met DSM-IV criteria for schizophrenia $(n=182)$ or schizoaffective disorder $(n=56)$ were enrolled as part of a larger longitudinal study investigating the course of cognitive and functional status. Subjects in this study were part of our previously published reports on the correlation between specific neuropsychological measures and functional outcome (Bowie et al., 2006, 2007). Exclusion criteria included a primary DSM-IV Axis I diagnosis other than schizophrenia or schizoaffective disorder with or without past substance disorders, Mini-Mental State Examination (MMSE; Folstein et al., 1975) score $<18$, Wide Range Achievement Test 3rd Edition (WRAT-3; Jastak \& Wilkinson, 1994) reading grade-equivalent $\leq 6$, or med- 
ical illnesses that might interfere with the assessment of cognitive functioning. The Comprehensive Assessment of Symptoms and History (CASH; Andreasen et al., 1992) was completed by trained research assistants and diagnosis was confirmed by a senior clinician. Patient data were used in these analyses only if they had case managers available as informants for real-world functional status ratings. Subjects were also required to demonstrate continued illness at the time of recruitment, as evidenced by meeting at least one of three criteria: (1) an inpatient admission for psychosis in the past two years; (2) an emergency room visit for psychosis in the past two years; or (3) a score on the PANSS positive symptoms items delusions, hallucinations, or conceptual disorganization of 4 (moderate) or more at the time of their baseline assessment. Outpatient status was defined as living outside of any institutional setting, including a nursing home.

All subjects were receiving treatment with secondgeneration antipsychotic medications at the time of the assessment. Recruitment was conducted at a VA or State Hospital or academic medical center and each subject received $\$ 50$ for their participation. After the testing procedures were fully explained, subjects signed a written informed consent form approved by the institutional review board at each site, where ethical approval was obtained.

\section{Measures}

All subjects completed the test battery in a fixed order. Screening measures included global cognition with the MMSE (Folstein et al., 1975) and estimated premorbid functioning with the WRAT-3 (Jastak \& Wilkinson, 1994) Reading subtest. These measures were followed by a functional skills assessment, a cognitive test battery, and a symptom interview. All interviewers received extensive training in performing all assessments and every three months their performance was evaluated through re-rating of training tapes, dual-ratings of the functional status measures with a senior staff, and quality assurance assessments of all testing.

\section{Performance-based measures of functional capacity}

The UCSD Performance-Based Skills Assessment (UPSA; Patterson et al., 2001a) was designed to directly evaluate the ability to perform everyday tasks that are considered necessary for independent community functioning. This test uses standardized role-playing situations to evaluate skills in different functional domains. In this study, 4 derived domains of the UPSA were used: comprehension/planning (e.g., organizing outings to the beach or zoo), finance (e.g., counting change, paying bills), transportation (e.g., using public transportation), and communication (e.g., using the telephone, rescheduling medication appointments). We excluded the household chores subtest because the analogue kitchen required was not portable enough to be used at field sites. We then re-standardized the scores to a 100point scale, like the original 5-subtest UPSA, thus allowing comparisons to previous results. This modified version was used in our previous reports with the UPSA (Bowie et al., 2005, 2006; McClure et al., 2007). Other studies have used modified versions like the two-subscale UPSA-Brief, which only includes the communication and financial subtests, and shows high correlation with the full version of the UPSA ( $r=.91)$ with excellent convergent and discriminant validity (Mausbach et al., 2007).

The Social Skills Performance Assessment (SSPA; Patterson et al., 2001b) is a social role-play task in which the subject initiates and maintains a conversation in two 3-minute role-play tasks: greeting a new neighbor and calling a landlord to request a repair for a leak that has gone unfixed. These sessions were audiotaped and scored by a trained rater who was unaware of diagnosis (patient or HC as part of a larger study) and all other data. Dimensions of social skills scored include fluency, clarity, focus, negotiation ability, persistence, and social appropriateness. These raters were trained to the gold standard ratings of the instrument developers, with an Intraclass Correlation Coefficient (ICC) of .86 and high inter-rater reliability was maintained at three months $(I C C=.87)$. The mean of the ratings on these variables across the two measures was used in this study.

\section{Real-world functional performance}

The Specific Level of Function Scale (SLOF; Schneider \& Streuening, 1983) was used to examine everyday functioning. This scale is a 43-item observer-rated report of behavior and functioning on the following domains: Physical Functioning (e.g., vision, hearing), Personal Care (e.g., eating, grooming), Interpersonal Relationships (e.g., initiating, accepting and maintaining social contacts), Socially Acceptability (e.g., verbal and physical abuse, repetitive behaviors), Participation in Community Activities (e.g., shopping, using telephone, paying bills, use of leisure time), and Work Skills (e.g., employable skills, punctuality). Ratings are made on a 5 -point Likert scale $(1=$ poor performance, $5=$ high performance) by a caseworker based on the amount of assistance that the patient requires to perform real-world skills or frequency of the behavior. All informants indicated that they knew the patient at least "very well" on the SLOF's 5-point Likert scale. The scale has excellent interrater reliability, factorial validity, and internal consistency (Schneider \& Streuening, 1983) and has been previously shown to be related to NP performance and scores on functional capacity measures (Bowie et al., 2006). Informants were unaware of the subject's performance on any cognitive or performance based measures or the symptom ratings. Interviewers completed and scored all aspects of assessment independently of the case managers' SLOF ratings.

To evaluate general elements of functional outcome, we used a categorical ranking of milestone achievements based on items from the CASH, which derived from a combination of self-report, case manager, and chart data. Independent living status was determined by whether the patient 
lived in restrictive or supported housing, as well as whether the patient was at least partially financially responsible for the residence. Patients were classified as living in restricted housing, living independently but not financially supporting the residence, or living independently and financially supporting the residence. Current work status was classified as unemployed, retired, or employed at least part time. In order to be considered retired, there must be a history of employment that terminated as a voluntary decision or after qualifying for pension. Marital status was classified as married or widowed, divorced or separated, or never married.

\section{Cognitive assessment}

Neuropsychological tests were selected to represent diverse cognitive domains that were previously shown to be the most consistently correlated with functional skills (Green, 1996; Green et al., 2000, Harvey, 2004). These tests included the Trail Making Test Parts A and B (Reitan \& Wolfson, 1993), short and long delay recall, learning trials $1-5$, and recognition from the Rey Auditory Learning Test (RAVLT; Morris et al., 1989), verbal fluency (Spreen \& Strauss, 1998), animal naming (Spreen \& Strauss, 1998), Stroop Color and Word Test interference condition (Golden, 1978), the Consortium to Establish a Registry for Alzheimer's Disease (CERAD) Constructional Praxis test (Morris et al., 1989), and the Digit Span, Letter-Number-Sequencing, and Digit Symbol Coding subtests of the Wechsler Adult Intelligence Scale, 3rd edition (WAIS-III; Psychological Corporation, 1998).

All raw scores on the NP tests were converted to age, education, and gender corrected standardized $(Z)$ scores and $T$-scores from published norms. The Global Deficit Score (GDS; Carey et al., 2004)) was used to identify NP normality and impairment. This approach is useful because it weights the NP data in a similar manner to clinical ratings by considering both the number and the severity of deficits in an individual's performances throughout the test battery, giving relatively less weight to performance within and above normal limits (Heaton et al., 1994, 1995). The GDS approach begins by converting $T$-scores to deficit scores that reflect presence and severity of impairment. $T$-scores greater than 40 represent no impairment (deficit score $=0$ ); whereas a deficit score of 1 reflects mild impairment $(T$-score $=39$ to 35); 2 reflects mild to moderate impairment $(T$-score $=34$ to 30 ); 3 reflects moderate impairment ( $T$-score $=29$ to $25) ; 4$ reflects moderate to severe impairment $(T$-score $=$ 24 to 20 ); and 5 reflects severe impairment $(T$-score $<20)$. After $T$-scores were converted to deficit scores, a composite RAVLT deficit score was calculated by using the mean of the four RAVLT subtest deficit scores to represent verbal memory, and a composite deficit score that included verbal fluency and animal naming (i.e., category fluency) was calculated to represent the fluency domain. Deficit scores on all tests were then averaged to create the GDS. In previous studies a GDS $\geq 0.5$ has accurately predicted expert clinical ratings of overall impairment (Carey et al., 2004). A GDS at this cutoff indicates that, on average, an individual was mildly impaired on half of the NP test measures in the battery or quite impaired on only one measure while performing quite well on the rest of the battery. As such, the NP normal group was composed of patients who performed within normal limits on the neuropsychological tests, but might not be neuropsychologically intact. The GDS method appears to be relatively unaffected by modifications in test batteries (Heaton et al., 1994; Carey et al., 2004).

\section{Symptom assessment}

Severity of schizophrenia symptoms was assessed using the Positive and Negative Syndrome Scale (PANSS; Kay et al., 1987). This is a 30-item scale with 7 items measuring positive symptoms, 7 items measuring negative symptoms, and 16 items measuring general aspects of psychopathology and is completed after a structured interview. Each item is scored on a scale ranging from 1 (absent) to 7 (extreme), with item ratings incorporating the behavioral effects of symptoms as well as their severity. Raters were trained to adequate reliability with ICC's from .86 to .92 . Specific items from the PANSS were selected by the Remission in Schizophrenia Working Group (Andreasen et al., 2005) for consideration as criteria for cross-sectional remission in schizophrenia to map the "A" criteria for schizophrenia specified in DSMIV. Specifically, items included delusions, unusual thought content, hallucinatory behavior, conceptual disorganization, mannerisms/posturing, blunted affect, social withdrawal, and lack of spontaneity. Thus, this classification implicates the absence of an active phase of illness, rather than an abatement of all psychiatric symptoms. Following the criteria proposed by Andreasen et al. (2005), a cutoff score of three ("mild") or less on each of these items was used for designation of cross-sectional symptom remission. Because of the cross-sectional design of the study, the current classification did not include a remission maintenance of over a 6-month period, as suggested by Andreasen et al. (2005).

\section{Statistical Analyses}

Two (NP normal and NP impaired) by two (symptom remission and no remission) analyses of covariance (ANCOVA) were conducted to examine differences between NP normal and remitted patients on the functional competence and realworld outcome measures, as well as the interaction of these two classifications. Education was entered as a covariate in these analyses to account for the affect of group differences on premorbid abilities. Performance on the WRAT was correlated $(r=.44, p<.01)$ with education, and thus was not used as an additional covariate. In addition to examining NP and remission status on functional competence and realworld outcome measures, we also examined these two variables in terms of their prevalence in cases who met various functional milestones, using the Chi-square test to compare these prevalences across these two dichotomous classifications. All analyses were performed using SPSS, version 14. 


\section{RESULTS}

\section{Sample Characteristics}

Two hundred and thirty-eight patients participated in the current study. Of the entire sample, eight patients could not be classified with symptom status because of missing PANSS data. Based on the classification system, as shown in Fig. 1, $18.5 \%(n=44)$ of the sample was identified as NP normal, $46.6 \%(n=111)$ was in cross-sectional symptomatic remission, and $8.8 \%(n=21)$ was both NP normal and remitted. The rate of normality found in the current sample is comparable to the rates (25\%) typically reported in other studies.

Demographic characteristics are presented in Table 1 and descriptive data for the neuropsychological and symptom measures is shown in Table 2. $11.1 \%$ of the sample did not report data on ethnicity and $2.1 \%$ of the sample did not report levels of education. All patients were receiving atypical antipsychotic medication, including $8.4 \%$ who were receiving clozapine. Age did not differ as a function of NP status, $F(1,225)=1.37, p>.05$, symptom status, $F(1,225)=$ $.73, p>.05)$ or the interaction of NP and symptom status, $F(1,225)=.41, p>.05)$. Although there were no differences in gender composition between the four subgroups, $\chi^{2}(3)=5.11, p>.05$, the composition of ethnicity significantly differed between groups, $\chi^{2}(9)=18.74, p<.05$, with a relatively greater proportion of minorities in the NP impaired groups compared to the NP normal groups.

Further, patients demonstrating NP normality had higher education compared to patients with impaired NP, $F(1,217)=11.28, p<.01$. Level of education was not significantly different across symptom status, $F(1,217)=$ $.03, p>.05$, nor was there an interaction effect, $F(1,217)=$ $.00, p>.05$. Similarly, patients with NP normality per-

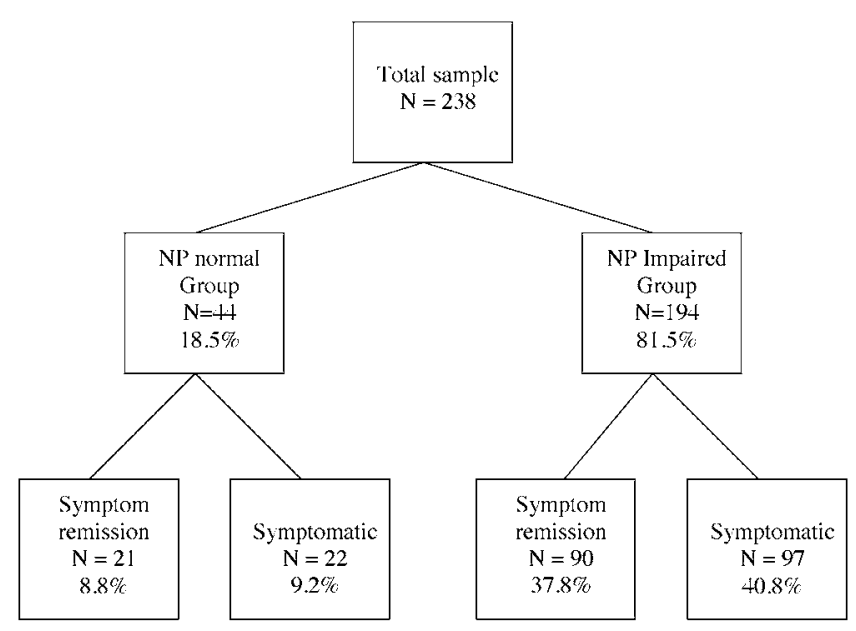

Fig. 1. Sample composition of patients with and without neuropsychological normality and cross-sectional symptom remission. Note: Missing data on the positive and negative syndrome scale lead to 8 less patients in the symptom remission and symptomatic groups. formed significantly better on the WRAT compared to patients with impaired NP, $F(1,226)=18.23, p<.01$, whereas there were no group differences between symptomatic and remitted patients, $F(1,226)=.18, p>.05$, and there was no interaction between NP and symptom status, $F(1,226)=.46, p>.05$. The duration of illness did not differ as a function of NP status, $F(1,226)=.07, p>.05$, symptom status, $F(1,226)=.52, p>.05$, or NP and symptom status interaction, $F(1,226)=1.00, p>.05$.

\section{Functional Competence and Everyday Outcomes as a Function of NP Normality and Symptom Remission}

Functional competence and ratings of everyday outcome data were analyzed using a series of two-by-two factorial ANCOVAs with NP status (NP normal and NP impaired) and cross-sectional symptom status (remitted or not remitted) as between-subjects variables and education as a covariate to account for premorbid functioning. Descriptive statistics are presented in Table 3. The ANCOVA for the UPSA total score revealed a significant main effect for NP status, $F(1,216)=23.69, p<.01$, partial $\eta^{2}=.10$, such that NP normal patients demonstrated better everyday living skills as compared to NP impaired patients. However, the main effect for symptom status, $F(1,216)=1.23, p>$ .05 , and the NP by symptom status interaction, $F(1,216)=$ $.52, p>.05$, were non-significant for the UPSA total score. Similarly, the ANCOVA for the SSPA total score revealed a significant main effect for NP status, $F(1,213)=5.94$, $p<.05$, partial $\eta^{2}=.03$, with NP normal patients showing better social competence compared to NP impaired patients, but there was no significant main effect for symptom status, $F(1,213)=1.54, p>.05$, or NP by symptom status interaction, $F(1,213)=2.75, p>.05$. As with functional competence, the ANCOVA for the SLOF total score revealed a significant main effect for NP status, $F(1,176)=$ $5.54, p<.05$, partial $\eta^{2}=.03$, with NP normal patients being less impaired than NP impaired patients on ratings of real-world function. The main effect for symptom status and the NP by symptom status interaction were not significant (all $p$ 's $>.05$ ).

To ensure that these results are not artifacts of dichotomizing variables that are typically viewed as continuous, Spearman correlations were performed on composite NP performance, total symptom severity, and functional outcomes. The GDS was negatively correlated with the UPSA $(r=-.64, p<.01)$, SSPA $(r=-.37, p<.01)$, and SLOF $(r=-.26, p<.01)$. In contrast, the PANSS was not significantly correlated with the UPSA $(r=-.09, p>.05)$ and has a small but significant correlation with the SSPA $(r=-.19, p<.05)$ and SLOF $(r=-.14, p<.05)$. These findings suggest that the relationship of daily functioning to neuropsychological performance and symptom severity is the same whether the analysis involved dichotomous or continuous variables. 
Table 1. Demographics characteristics of the current sample

\begin{tabular}{|c|c|c|c|c|c|c|c|c|}
\hline \multirow[b]{3}{*}{ Variable } & \multicolumn{8}{|c|}{ Group } \\
\hline & \multicolumn{2}{|c|}{$\begin{array}{l}\text { NPN-remitted } \\
\quad(N=21)\end{array}$} & \multicolumn{2}{|c|}{$\begin{array}{l}\text { NPI-remitted } \\
\quad(N=22)\end{array}$} & \multicolumn{2}{|c|}{$\begin{array}{l}\text { NPN-symptomatic } \\
\quad(N=90)\end{array}$} & \multicolumn{2}{|c|}{$\begin{array}{l}\text { NPI-symptomatic } \\
\qquad(N=97)\end{array}$} \\
\hline & M & SD & M & SD & M & SD & M & SD \\
\hline Age (years) & 59.0 & 9.1 & 55.9 & 10.4 & 56.4 & 9.1 & 55.5 & 9.8 \\
\hline Illness chronicity (years) & 30.4 & 10.5 & 28.1 & 12.2 & 27.2 & 9.9 & 28.6 & 10.0 \\
\hline Education (years) & 13.9 & 2.2 & 12.3 & 3.0 & 13.9 & 2.5 & 12.4 & 2.4 \\
\hline \multirow[t]{2}{*}{ WRAT score (raw) } & 49.1 & 6.9 & 44.0 & 8.1 & 49.5 & 5.7 & 42.5 & 9.4 \\
\hline & $\mathrm{N}$ & $\%$ & $\mathrm{~N}$ & $\%$ & $\mathrm{~N}$ & $\%$ & $\mathrm{~N}$ & $\%$ \\
\hline $\operatorname{Sex}(\%$ male $)$ & 11 & 52.4 & 68 & 75.6 & 17 & 77.3 & 72 & 74.2 \\
\hline \multicolumn{9}{|l|}{ Ethnicity } \\
\hline Caucasian & 15 & 78.9 & 49 & 59.8 & 17 & 85.0 & 42 & 50.6 \\
\hline African American & 4 & 21.1 & 23 & 28.0 & 3 & 15.0 & 37 & 44.6 \\
\hline American Indian & 0 & 0.0 & 2 & 2.4 & 0 & 0 & 0 & 0.0 \\
\hline Other & 0 & 0.0 & 8 & 9.8 & 0 & 0.0 & 4 & 4.8 \\
\hline
\end{tabular}

Note. NPN = neuropsychologically normal; NPI = neuropsychologically impaired; missing data on education and ethnicity lead to N's less than the complete sample.

\section{Real-World Milestones as a Function of NP Normality}

The prevalence of three milestone indicators, including independent residential status, current work and marital status, as a function of NP status is presented in Fig. 2. Independent residential status differed by group, $\chi^{2}(2)=6.46, p<$ .05. This effect seems to be driven by the tendency for patients who were NP normal to be more likely to be living independently and financially responsible for their dwelling, whereas patients who were NP impaired were more likely to be living in a restrictive setting. Both current work status, $\chi^{2}(2)=.53, p>.05$, and current marital status did not differ by NP status, $\left.\chi^{2}(2)=.62, p>.05\right)$.

\section{Real-World Milestones as a Function of Symptomatic Remission}

Independent residential status, $\left.\chi^{2}(2)=.58, p>.05\right)$, current work status, $\chi^{2}(2)=2.0, p>.05$, and current marital status did not differ by cross-sectional symptomatic remission status, $\left.\chi^{2}(2)=.18, p>.05\right)$.

Table 2. Neuropsychological performance and symptom profiles

\begin{tabular}{|c|c|c|c|c|}
\hline \multirow[b]{3}{*}{ Measure ( $T$ score $)$} & \multicolumn{4}{|c|}{ Group } \\
\hline & NPN-remitted & NPI-remitted & NPN-symptomatic & NPI-symptomatic \\
\hline & $\mathrm{M}(\mathrm{SD})$ & $\mathrm{M}(\mathrm{SD})$ & $\mathrm{M}(\mathrm{SD})$ & $\mathrm{M}(\mathrm{SD})$ \\
\hline Trail Making Test, Part A & $46.4(6.8)$ & $20.8(25.2)$ & $46.2(7.2)$ & $11.1(32.1)$ \\
\hline Trail Making Test, Part B & $46.6(6.6)$ & $14.7(25.6)$ & $46.3(6.4)$ & $9.1(26.5)$ \\
\hline RAVLT composite score & $44.4(11.8)$ & $34.5(11.1)$ & $45.3(11.5)$ & $31.6(12.1)$ \\
\hline RAVLT Short Delay Recall & $45.7(12.7)$ & $35.3(10.4)$ & $45.8(14.8)$ & $33.1(10.6)$ \\
\hline RAVLT Long Delay Recall & $43.7(13.0)$ & $34.1(11.2)$ & $46.0(12.5)$ & $32.2(10.6)$ \\
\hline RAVLT Trials $1-5$ & $43.5(12.0)$ & $31.3(11.6)$ & $45.7(11.2)$ & $30.5(12.4)$ \\
\hline RAVLT Recognition & $44.6(15.8)$ & $37.3(22.1)$ & $43.5(17.3)$ & $30.4(25.0)$ \\
\hline Fluency composite score & $45.1(5.6)$ & $40.2(8.4)$ & $45.6(7.1)$ & $40.3(10.8)$ \\
\hline Verbal Fluency & $44.0(8.4)$ & $38.5(10.1)$ & $42.6(7.6)$ & $39.9(11.1)$ \\
\hline Animal Naming & $46.2(7.1)$ & $41.9(11.2)$ & $48.6(9.2)$ & $40.7(14.0)$ \\
\hline Stroop interference & $50.8(7.0)$ & $45.3(8.3)$ & $45.6(3.2)$ & $44.6(7.3)$ \\
\hline Constructional praxis & $51.0(5.7)$ & $42.3(9.5)$ & $47.4(6.9)$ & $42.4(8.6)$ \\
\hline Digit Span & $51.6(11.0)$ & $42.8(7.0)$ & $49.6(6.8)$ & $42.6(7.9)$ \\
\hline Letter Number Sequence & $49.8(8.9)$ & $35.8(8.4)$ & $48.1(6.6)$ & $35.7(9.8)$ \\
\hline Digit Symbol Coding & $45.8(7.0)$ & $33.8(6.4)$ & $43.7(6.1)$ & $33.3(5.8)$ \\
\hline PANSS total score (raw) & $43.4(9.1)$ & $46.2(9.0)$ & $64.1(13.2)$ & $64.5(13.4)$ \\
\hline
\end{tabular}

Note. NPN = neuropsychologically normal; NPI = neuropsychologically impaired; Scores are presented as $t$-scores, with a mean of 50 and an SD of 10 . 
Table 3. Functional capacity performance and everyday outcome rated by case managers as a function of neuropsychological and symptom status

\begin{tabular}{|c|c|c|c|c|c|c|c|c|}
\hline \multirow[b]{3}{*}{ Measure } & \multicolumn{8}{|c|}{ Group } \\
\hline & \multicolumn{2}{|c|}{$\mathrm{NP} ; ; 2 \mathrm{~N}$-remitted } & \multicolumn{2}{|c|}{ NPI-remitted } & \multicolumn{2}{|c|}{ NPN-symptomatic } & \multicolumn{2}{|c|}{ NPI-symptomatic } \\
\hline & M & SD & M & SD & M & $\mathrm{SD}$ & M & SD \\
\hline UPSA total score ${ }^{a}$ & 87.3 & 10.0 & 71.7 & 17.3 & 86.3 & 12.5 & 66.7 & 19.6 \\
\hline SSPA total score ${ }^{b}$ & 4.2 & 0.5 & 4.0 & 0.7 & 4.2 & 0.5 & 3.7 & 0.8 \\
\hline SLOF total score ${ }^{c}$ & 205.6 & 10.2 & 193.3 & 19.9 & 198.7 & 8.2 & 191.0 & 21.7 \\
\hline
\end{tabular}

Note. NPN = neuropsychologically normal; NPI = neuropsychologically impaired.

${ }^{a} U C S D$ Performance-Based Skills Assessment, with a possible range of 0-100.

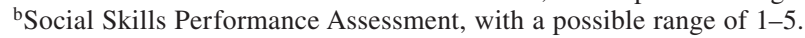

${ }^{\mathrm{c} S p e c i f i c ~ L e v e l ~ o f ~ F u n c t i o n i n g ~ S c a l e, ~ w i t h ~ a ~ p o s s i b l e ~ r a n g e ~ o f ~ 43-215 . ~}$

\section{DISCUSSION}

The purpose of this study is to examine the cross-sectional functional correlates of NP normality and symptom remission in schizophrenia in terms of both functional capacity and ratings of everyday behavior, and to evaluate more general elements of functional outcome and their relationship with NP normality and cross-sectional symptomatic remission. Several findings emerged. First, NP status contributed to group differences on functional skills, social competence, and ratings of everyday functioning, such that NP normal patients had better functional skills and ratings of real-world functioning compared to NP impaired patients. This effect was significant even after controlling for premorbid functioning. In contrast, neither cross-sectional symptom status nor the interaction between NP and symptom status differentiated functional outcome. Consistent with the existing literature on the association between NP performance and functional outcome (Green, 1996; Green et al., 2000), these results suggest that NP normality may be a better predictor of performance on measures of functional capacity compared to cross-sectional remission. These results also suggest that the classification of NP normality may provide a meaningful categorization for the concept of outcome that is in line with the recovery model emerging in the recent literature (Bellack, 2006).

The most important finding in this study is that NP normal cases still show deficits in several domains of everyday functioning milestones. Although NP normal cases have better living skills (i.e., capacity) than NP impaired cases, NP normal cases have an average impairment level on the UPSA total score that is still about one SD below the performance of healthy controls in previous studies (Patterson et al., 2001a). The rate of independence in residential status, although higher in the NP normal sample than the others, is still reduced compared to the general population expectation. Histories of employment and marriage were not different between the samples.

A host of external factors apart from cognition, outside the scope of the present study, are likely to affect functional outcome. For example, social stigma, lack of social support, and financial resources might be barriers for functional skills to generalize to performance in the real world (Bellack et al., 2007). Competitive employment among patients diagnosed with schizophrenia may be impeded by the potential adverse incentive of disability payments as
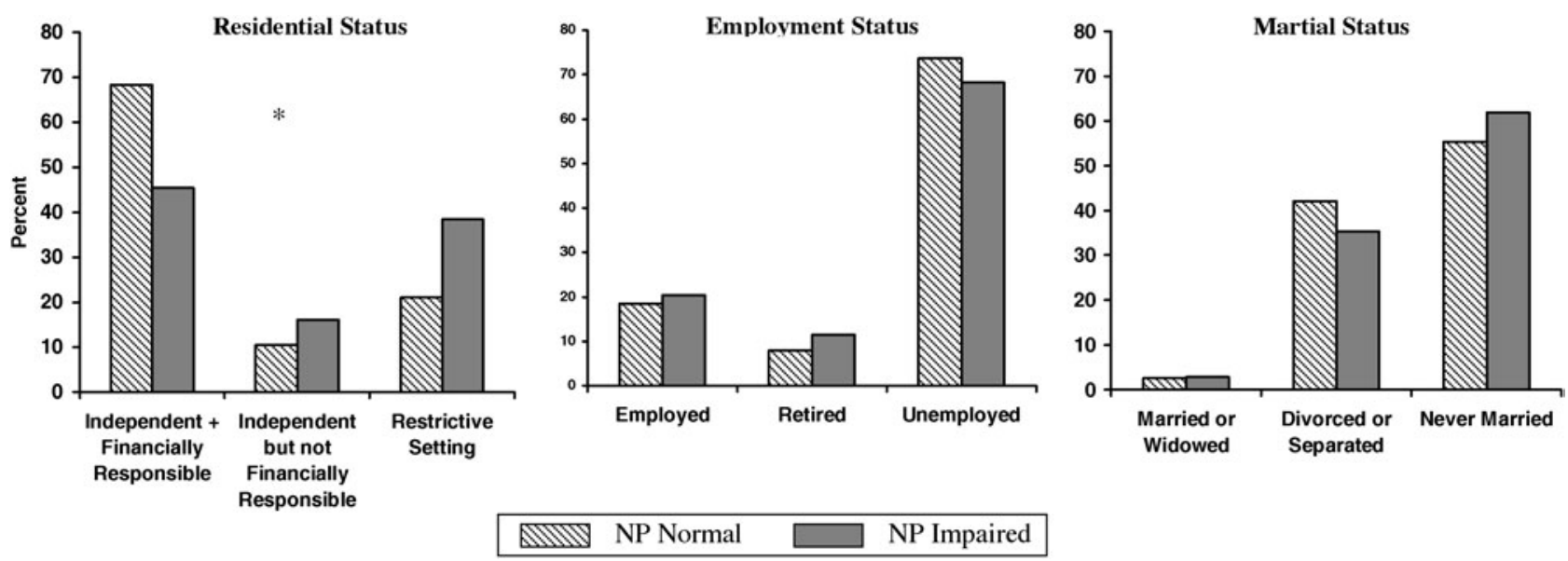

Fig. 2. Real-world milestones as a function of neuropsychological normality. ${ }^{*} p<.05$. 
well as ethnic factors, and may be promoted by the availability of rehabilitation services (Rosenheck et al., 2006). Additionally, social cognition has recently been found to be a potential mediator of the influence of cognitive impairments on everyday outcomes (Brekke et al., 2005; Sergi et al., 2007). Related to, but separate from social cognition are metacognitive processes, which have been argued to account for poor functional outcome in some cases despite intact cognitive abilities (Koren \& Harvey, 2006).

Of note, the amount of variance explained by NP normality was not as great as that seen in previous studies where the full range of NP performance was considered (e.g., Bowie et al., 2006; Green et al., 2000), suggesting that a dimensional approach that presents a range of NP performance may be more informative, in this domain, than a dichotomous classification system. This may be related to the fact that there is considerable variance in cognitive performance within the large group designated as impaired in their performance, which is not reflected in the dichotomous classification. Further, our classification of NP normality does not consider relative impairments within individuals diagnosed with schizophrenia. As noted by Wilk et al. (2005), even patients who appear to perform within normal limits are often still performing much more poorly than would be expected from their intellectual performance. Whereas a dimensional approach has its advantages, the goal of this study is to investigate the functional implications of a subgroup of patients with NP normality and findings provide support that this classification is clinically meaningful as NP status differentiates functional outcome.

Our data indicate that this is a high functioning schizophrenia sample, which may limit the generalizability of the current findings. This investigation is part of a larger project, with the overarching aim to track changes into late life in a sample of older, community-dwelling patients. Thus, our recruitment sample, whereas not generalizable to the entire range of this heterogeneous condition, is likely more representative of patients observed in mental health clinics.

Several limitations of this study should be addressed. One such limitation, which likely reduced the predictive power of symptomatic remission, is that our criteria for remission did not include the minimum 6-month duration suggested by Andreasen et al. (2005). The cross-sectional nature of this study raises the possibility that this sample is experiencing transient symptom reduction. A recent study by De Hert et al. (2007) indicated that sustained symptomatic remission was associated with functional gains. The contrast between these findings suggests that more research on the clinical and functional importance of sustained, as compared to cross-sectional, remission is required. As part of a larger longitudinal study, we will further examine the long-term functional outcome of sustained symptomatic remission as follow-up data become available. Despite utilizing a comprehensive neuropsychological battery to assess cognitive domains that were previously shown to be the most consistently impaired in schizophrenia, other areas such as sensory ability and visual memory were not included, which may have affected the classification of normality. Yet, the rate of normality in the current study is comparable to the rate typically found in other studies (Bryson et al., 1993; Kremen et al., 2000; Palmer et al., 1997; Rund et al., 2006). Although the small number of subjects identified as having NP normality and cross-sectional symptomatic remission is not surprising given the low base rate of NP normality in schizophrenia, it nevertheless may have lowered the power to detect functional outcomes associated with this group. Future studies would need to include a substantially larger sample in order to have sufficient power to systematically study patients with both NP normality and remission. Participation in competitive employment has been reported to be influenced by race in schizophrenia (Rosenheck et al., 2006), but in this study there were no differences in employment status as a function of NP status, despite the disproportionate number of minorities in this subgroup. Finally, measures of functional competence employed in this study are proxy measures of everyday functioning. As such, findings should be interpreted with caution, because performance on proxy measures may not translate one to one with performance in the community caused by the factors described earlier.

\section{ACKNOWLEDGMENTS}

This research was supported by NIMH Grant Number MH 63116 to Dr. Harvey, the Mt. Sinai Silvio Conte Neuroscience Center (NIMH MH 36692; KL Davis PI), and the VA VISN 3 MIRECC.

\section{REFERENCES}

Andreasen, N.C., Carpenter, W.T., Kane, J.M., Lasser, R.A., Marder, S.R., \& Weinberger, D.R. (2005). Remission in schizophrenia: Proposed criteria and rationale for consensus. American Journal of Psychiatry, 162, 441-449.

Andreasen, N.C., Flaum, M., \& Arndt, S. (1992). The comprehensive assessment of symptoms and history (CASH): An instrument for assessing psychopathology and diagnosis. Archives of General Psychiatry, 49, 615-623.

Bellack, A.S. (2006). Scientific and consumer models of recovery in schizophrenia: Concordance, contrasts, and implications. Schizophrenia Bulletin, 32, 432-442.

Bellack, A.S., Green, M.F., Cook, J.A., Fenton, W., Harvey, P.D., Heaton, R.K., Laughren, T., Leon, A.C., Mayo, D.J., Patrick, D.L., Patterson, T.L., Rose, A., Stover, E., \& Wykes, T. (2007). Assessment of community functioning in people with schizophrenia and other severe mental illness: A white paper based on an NIMH-sponsored workshop. Schizophrenia Bulletin, 33, 805-822.

Bowie, C.R., Leung, W.W., Reichenberg, A., McClure, M.M., Patterson, T.L., Heaton, R.K., Harvey, P.D. (in press). Predicting schizophrenia patients' real world behavior with specific neuropsychological and functional capacity measures. Biological Psychiatry.

Bowie, C.R., Reichengerg, A., Patterson, T.L., Heaton, R.K., \& Harvey, P.D. (2006). Determinants of real world functional performance in schizophrenia: Correlations with cognition, functional capacity, and symptoms. American Journal of Psychiatry, 163, 418-425. 
Bowie, C.R., Serper, M.R., Riggio, S., \& Harvey, P.D. (2005). Neurocognition, symptomatology, and functional skills in older alcohol-abusing schizophrenia patients. Schizophrenia Bulletin, 31, 175-182.

Bowie, C.R., Twamley, E.W., Anderson, H., Halpern, B., Patterson, T.L., \& Harvey, P.D. (2007). Self-assessment of functional status in schizophrenia. Journal of Psychiatry Research, 41, 1012-1018.

Bozikas, V.P., Kosmidis, M.H., Kiosseoglou, G., \& Karavatos, A. (2006). Neuropsychological profile of cognitively impaired patients with schizophrenia. Comprehensive Psychiatry, 47, 136-143.

Brekke, J., Kay, D.D., Lee, K.S., \& Green, M.F. (2005). Biosocial pathways to functional outcome in schizophrenia. Schizophrenia Research, 80, 213-225.

Bryson, G.J., Silverstein, M.L., Nathan, A., \& Stephen, L. (1993). Differential rate of neuropsychological dysfunction in psychiatric disorders: Comparison between Halstead-Reitan and LuriaNebraska batteries. Perceptual and Motor Skills, 76, 305306.

Buchanan, R.W., Davis, M., Goff, D., Green, M.F., Keefe, R.S., Leon, A.C., Nuechterlein, K.H., Laughren, T., Levin, R., Stover, E., Fenton, W., \& Marder, S.R. (2005). A summary of the FDANIMH-MATRICS workshop on clinical trial design for neurocognitive drugs for schizophrenia. Schizophrenia Bulletin, 31, 5-19.

Carey, C.L., Woods, S.P., Gonzalez, R., Conover, E., Marcotte, T.D., Grant, I., Heaton, R.K., \& the HNRC Group. (2004). Predictive validity of global deficit scores in detecting neuropsychological impairment in HIV infection. Journal of Clinical and Experiential Neuropsychology, 26, 307-319.

De Hert, M., van Winkel, R., Wampers, M., Kane, J., van Os, J., \& Peuskens, J. (2007). Remission criteria for schizophrenia: Evaluation in a large naturalistic cohort. Schizophrenia Research, $92,68-73$.

Evans, J.D., Heaton, R.K., Paulsen, J.S., Palmer, B.W., Patterson, T., \& Jeste, D.V. (2003). The relationship of neuropsychological abilities to specific domains of functional capacity in older schizophrenic patients. Biological Psychiatry, 53, 422-430.

Folstein, M.F., Folstein, S.E., \& McHugh, P.K. (1975). Minimental state: A practical method for grading the cognitive state of patients for the clinician. Journal of Psychiatric Research, 12, 189-198.

Frank, E., Prien, R.F., Jarrett, R.B,, Keller, M.B., Kupfer, D.J., Lavori, P.W., Rush, A.J., \& Weissman, M.M. (1991). Conceptualization and rationale for consensus definitions of terms in major depressive disorder: Remission, recovery, relapse, and recurrence. Archives of General Psychiatry, 48, 851-885.

Golden, C.J. (1978). Stroop Color and Word Test: A manual for clinical and experimental uses. Wood Dale, IL: Stoelting.

Goldstein, G. (1994). Neurobehavioral heterogeneity in schizophrenia. Archives of Clinical Neuropsychology, 9, 265-276.

Green, M.F. (1996). What are the functional consequences of neurocognitive deficits in schizophrenia? American Journal of Psychiatry, 153, 321-330.

Green, M.F., Kern, R.S., Braff, D.L., \& Mintz, J. (2000). Neurocognitive deficits and functional outcome in schizophrenia: Are we measuring the "right stuff?" Schizophrenia Bulletin, 26, 119-136.

Green, M.F., Kern, R.S., \& Heaton, R.K. (2004). Longitudinal studies of cognition and functional outcome in schizophrenia: Implications for MATRICS. Schizophrenia Research, 72, 41-51.
Hafner, H. \& an der Heiden, W. (2003). Course and outcome of schizophrenia. In S.R. Hirsch \& D.R. Weinberger (Eds.), Schizophrenia, 2nd ed. (pp. 101-141). Malden, MA: Blackwell Publishing.

Harvey, P.D. (2004). Schizophrenia in late life: Aging effects on symptoms and course of illness. Washington DC: American Psychological Association.

Harvey, P.D., Green, M.F., McGurk, S.R., \& Meltzer, H.Y. (2003). Changes in cognitive functioning with risperidone and olanzapine treatment: A large-scale, double blind, randomized study. Psychopharmacology, 169, 404-411.

Harvey, P.D., Howanitz, E., Parrella, M., White, L., Davidson, M., Mohs, R.C., Hoblyn, J., \& Davis, K.L. (1998). Symptoms, cognitive functioning, and adaptive skills in geriatric patients with lifelong schizophrenia: A comparison across treatment sites. American Journal of Psychiatry, 155, 1080-1086.

Harvey, P.D., Patterson, T.L., Potter, L.S., Zhong, K., \& Brecher, M. (2006). Improvement in social competence with short-term atypical antipsychotic treatment: A randomized, double-blind comparison of Quetiapine versus Risperidone for social competence, social cognition, and neuropsychological functioning. American Journal of Psychiatry, 163, 1918-1925.

Harvey, P.D., Siu, C.O., \& Romano, S. (2004). Randomized, controlled, double-blind multicenter comparison of the cognitive effects of ziprasidone versus olanzapine in acutely ill inpatients diagnosed with schizophrenia or schizoaffective disorder. Psychopharmacology, 172, 324-332.

Heaton, R.K., Grant, I., Butters, N., White, D.A., Kirson, D., Atkinson, J.H., McCutchan, J.A., Taylor, M.J., Kelly, M.D., Ellis, R.J., Wolfson, T., Velin, R., Marcotte, T.D., Hesselink, J.R., Jernigan, T.L., Chandler, J., Wallace, M., Abramson, I., \& the HNRC Group. (1995). The HNRC 500-Neuropsychology of HIV infection at different disease stages. Journal of International Neuropsychological Society, 1, 231-251.

Heaton, R.K., Paulsen, J.S., McAdams, L.A., Kuck, J., Zisook, S., Braff, D., Harris, J., \& Jeste, D.V. (1994). Neuropsychological deficits in schizophrenics. Relationship to age, chronicity, and dementia. Archives of General Psychiatry, 51, 469-476.

Hogarty, G.E., Flesher, S., Ulrich, R.F., Carer, M., Greenwald, D., Pogue-Geile, M., Kechavan, M., Cooley, S., DiBarry, A.L., Garrett, A., Parepally, H., \& Zoretich, R. (2004). Cognitive enhancement therapy for schizophrenia: Effects of a 2-year randomized trial on cognition and behavior. Archives of General Psychiatry, 61, 866-876.

Jastak, S. \& Wilkinson, G. (1994). The Wide Range Achievement Test, 3rd ed. San Antonio, TX: The Psychological Corporation.

Kay, S.R., Fiszbein, A., \& Opler, L.A. (1987). The Positive and Negative Syndrome Scale (PANSS) for schizophrenia. Schizophrenia Bulletin, 13, 261-276.

Keefe, R.S.E., Frecka,, E., Apter, S., Davidson, M., Macaluso, J.M., Hirschowitz, J., \& Davis, K.L. (1996). Clinical characteristics of kraepelinian schizophrenia: A replication and extension of previous findings. American Journal of Psychiatry, 153, 806-811.

Keefe, R.S., Seidman, L.J., Christensen, B.K., Hamer, R.M., Sharma, T., Sitskoorm, M.M., Lewine, R.R., Yurgelun-Todd, D.A., Gur, R.C., Tohen, M., Tollefson, G.D., Sanger, T.M., \& Lieberman, J.A. (2004). Comparative effects of atypical and conventional antipsychotic drugs on neurocognition in firstepisode psychosis: A randomized, double-blind trial of olanzapine versus low doses of haloperidol. American Journal of Psychiatry, 161, 985-995. 
Koren, D. \& Harvey, P.D. (2006). Closing the gap between cognitive performance and real-world functional outcome in schizophrenia: The importance of metacognition. Current Psychiatry Reviews, 2, 198-198.

Kremen, W.S., Seidman, L.J., Faraone, S.V., Toomey, R., \& Tsuang, M.T. (2000). The paradox of normal neuropsychological function in schizophrenia. Journal of Abnormal Psychology, 109, 743-752.

Marder, S.R. \& Fenton, W. (2004). Measurement and treatment research to improve cognition in schizophrenia: NIMH MATRICS initiative to support the development of agents for improving cognition in schizophrenia. Schizophrenia Research, 72, 5-9.

Mausbach, B.T., Harvey, P.D., Goldman, S.R., Jeste, D.V., \& Patterson, T.L. (2007). Development of a brief scale of everyday functioning in persons with serious mental illness. Schizophrenia Bulletin, 33, 1364-1372.

McClure, M.M., Bowie, C.R., Patterson, T.L., Heaton, R.K., Weaver, C., Anderson, H., \& Harvey, P.D. (2007). Correlations of functional capacity and neuropsychological performance in older patients with schizophrenia: Evidence for specificity of relationship? Schizophrenia Research, 89, 330-338.

McGurk, S.R., Mueser, K.T., \& Pascaris, A. (2005). Cognitive training and supported employment for persons with severe mental illness: One-year results from a randomized controlled trial. Schizophrenia Bulletin, 31, 898-909.

Morris, J.C., Heyman, A., Mohs, R.C., Hughes, J.P., Van Belle, G., Fillenbaum, G., Mellitis, E.D., \& Clark, C. (1989). The Consortium to Establish a Registry for Alzheimer's Disease (CERAD). Part I. Clinical and neuropsychological assessment of Alzheimer's disease. Neurology, 39, 1159-1165.

Mueser, K.T. (2000). Cognitive functioning, social adjustment, and long-term outcome in schizophrenia. In T. Sharma \& P.D. Harvey (Eds.), Cognition in Schizophrenia (pp. 157-177). New York: Oxford University Press.

Nuechterlein, K.H., Miklowitz, D.J., Ventura, J., Gitlin, M.J., Stoddard, M., \& Lukoff, D. (2006). Classifying episodes in schizophrenia and bipolar disorder: Criteria for relapse and remission applied to recent-onset samples. Psychiatry Research, 144, 153-166.

Palmer, B.W., Heaton, R.K., Gladsjo, J.A., Evans, J.D., Patterson, T.L., Golshan, S., \& Jeste, D.V. (2002). Heterogeneity in functional status among older outpatients with schizophrenia: Employment history, living situation, and driving. Schizophrenia Research, 55, 205-215.

Palmer, B.W., Heaton, R.K., Paulsen, J.S., Kuck, J., Braff, D., Harris, M.J., Zisook, S., \& Jeste, D.V. (1997). Is it possible to be schizophrenic yet neuropsychologically normal? Neuropsychology, 11, 437-446.

Patterson, T.L., Goldman, S., McKibbin, C.L., Hughs, T., \& Jeste, D.V. (2001a). UCSD performance-based skills assessment: Development of a new measure of everyday functioning for severely mentally ill adults. Schizophrenia Bulletin, 27, 235-245.

Patterson, T.L., Moscona, S., McKibbin, C.L., Davidson, K., \&
Jeste, D.V. (2001b). Social skills assessment performance assessment among older patients with schizophrenia. Schizophrenia Research, 48, 351-360.

Psychological Corporation (1998). WAIS-III and WMS-III Technical Manual. San Antonio, TX: Author.

Reitan, R.M. \& Wolfson, D. (1993). The Halstead-Reitan neuropsychological test battery: Theory and clinical interpretation. 2nd edition. Tucson, AZ: Neuropsychology Press.

Rosenheck, R., Leslie, D., Keefe, R., McEvoy, J., Swartz, M., Perkins, D., Stroup, S., Hsiao, J.K., Lieberman, J., \& CATIE Study Investigators Group. (2006). Barriers to employment for people with schizophrenia. American Journal of Psychiatry, 163, 411-417.

Rund, B.R., Sundet, K., Asbjornsen, A., Egeland, J., Landro, N.I., Lund, A., Roness, A., Stordal, K.I., \& Hugdahl, K. (2006). Neuropsychological test profiles in schizophrenia and nonpsychotic depression. Acta Psychiatrica Scandinavica, 113, 350-359.

Schneider, L.C. \& Streuening, E.L. (1983). SLOF: A behavioral rating scale for assessing the mentally ill. Social Work Research and Abstracts, 6, 9-21.

Sergi, M.J., Rassovsky, Y., Widmark, C., Reist, C., Erhart, S., Braff, D.L., Marder, S.R., \& Green, M.F. (2007). Social cognition in schizophrenia: Relationships with neurocognition and negative symptoms. Schizophrenia Research, 90, 316-324.

Smith, T.E., Hull, J.W., Romanelli, S., Fertuck, E., \& Weiss, K.A. (1999). Symptoms and neurocognition as rate limiters in skills training for psychotic patients. American Journal of Psychiatry, 156, 1817-1818.

Spreen, O. \& Strauss, E.A. (1998). Compendium of neuropsychological tests and norms, (2nd ed.). New York: Oxford University Press.

Torrey, E.F., Bowler, A.E., Taylor, E.H., \& Gottesman, I.I. (1994). Schizophrenia and manic-depressive disorder: The biological roots of mental illness as revealed by the landmark study of identical twins. New York: Basic Books.

Twamley, E.W., Doshi, R.R., Nayak, G.V., Palmer, B.W., Golshan, S., Heaton, R.K., Patterson, T.L., \& Jeste, D.V. (2002). Generalized cognitive impairments, ability to perform everyday tasks, and level of independence in community living situations of older patients with psychosis. American Journal Psychiatry, 159, 2013-2020.

van Os, J., Drukker, M., a Campo, J., Meijer, J., Bak, M., \& Delespaul, P. (2006). Validation of remission criteria for schizophrenia. American Journal of Psychiatry, 163, 2000-2002.

Wexler, B.E. \& Bell, M.D. (2005). Cognitive remediation and vocational rehabilitation for schizophrenia. Schizophrenia Bulletin, 31, 931-941.

Wilk, C.M., Gold, J.M., McMahon, R.P., Humber, K., Iannone, V.N., \& Buchanan, R.W. (2005). No, it is not possible to be schizophrenic yet neuropsychologically normal. Neuropsychology, 19, 778-786. 